\title{
Simulation of Valence EELS and Optical Response Functions from First Principles
}

\author{
V. J. Keast* R. W. Burgess* and D. P. Walton* \\ * School of Mathematical and Physical Sciences, The University of Newcastle, Callaghan, NSW \\ 2308, Australia
}

Recent years have seen an increase in the interest and application of the low-loss region $(<20 \mathrm{eV})$ in electron energy-loss spectroscopy (EELS). The spectral detail in this region is associated with excitations of the valence electrons and includes interband transitions and collective excitations. In addition, for many materials, an independent particle approximation for the excitations will be inadequate and quasi-particle or excitonic effects must be considered. For these reasons this socalled valence EELS can be quite complex and calculation of the spectra from first principles is important for their interpretation. The valence EELS has connections to optical response functions and there are a number of sophisticated approaches already available for the calculations of these; so the task is less daunting than it might first appear. However, the incident electric fields associated with light or with a fast electron are distinctly different from one another in their time and spatial dependence. This becomes particularly important for inhomogeneous materials.

The traditional approach to EELS, as first derived by Ritchie [1], starts by considering the total energy loss as the work done on the external charge, $\rho_{\text {ext }}(\mathbf{r}, t)$, (the incident electron with trajectory $\left.\mathbf{r}_{0}(\mathrm{t})\right)$ by the induced potential $V_{\text {ind }}(\mathbf{r}, t)$ :

$$
W=\left.\int_{-\infty}^{\infty} \rho_{\text {ext }}(\mathbf{r}, t) \nabla V_{\text {ind }}(\mathbf{r}, t) \cdot \mathbf{v}\right|_{\mathbf{r}=\mathbf{r}_{0}(t)} d t
$$

An expression for the induced potential is found, the temporal and spatial Fourier transforms of the induced potential and external charge are used and the energy loss probability can be determined by recognizing that the total energy loss is also given by:

$$
W=\int_{0}^{\infty} \hbar \omega \frac{d \sigma}{d \omega} d \omega
$$

An equivalent and arguably more revealing starting point is to instead consider the electrostatic potential energy of the induced charge, $\rho_{\text {ind }}(\mathbf{r}, t)$, due to the external potential $V_{\text {ext }}(\mathbf{r}, t)$.

$$
W=\int_{-\infty}^{\infty} \int_{-\infty}^{\infty} V_{\text {ext }}(\mathbf{r}, t) \frac{d}{d t} \rho_{\text {ind }}(\mathbf{r}, t) d t d \mathbf{r}
$$

The density response function (sometimes referred to as the reducible polarizability), $\chi\left(\mathbf{r}, \mathbf{r}^{\prime}, t, t^{\prime}\right)$, connects the induced charge to the external potential, and can be introduced to give.

$$
W=\int_{-\infty}^{\infty} \int_{-\infty}^{\infty} \int_{-\infty}^{\infty} \int_{-\infty}^{\infty} V_{e x t}(\mathbf{r}, t) \frac{d}{d t} \chi\left(\mathbf{r}, \mathbf{r}^{\prime}, t, t^{\prime}\right) V_{\text {ext }}\left(\mathbf{r}^{\prime}, t^{\prime}\right) d t d t^{\prime} d \mathbf{r} d \mathbf{r}^{\prime}
$$

This equation is more general as it can apply to any external potential, either that of light or an incident electron. In addition, the connection of EELS to calculation methods developed for optical spectra, which focus on first deriving $\chi\left(\mathbf{r}, \mathbf{r}^{\prime}, t, t^{\prime}\right)$, becomes more clear. In the trivial case of bulk materials and using the appropriate expressions for the external potentials it is possible to derive 
from Eqn. 4 the usual expressions for the energy-loss spectra $[I(\omega) \propto \operatorname{Im}(-1 / \varepsilon)]$ and optical absorption spectra $[(I(\omega) \propto \operatorname{Im}(\varepsilon)]$. Fig. 1 shows EEL spectra calculated for bulk LiF using Yambo [2], a code developed for many body calculations of optical spectra. Only when full quasiparticle corrections and excitonic effects are included are the experimental spectra reasonably well reproduced. Fig. 2 shows optical absorption spectra for clusters of 55 gold atoms of two different atomic arrangements. These were calculated using Octopus [3], a real-space time dependent density functional theory (TDDFT) code. As yet, the existing and readily available codes for calculation of optical properties, such as these, have not been adapted to calculate the spatially resolved EELS in inhomogeneous systems. However, as Eqn. 4 illustrates, the necessary modifications should not be difficult to implement.

[1] R.H. Ritchie, Phys. Rev. 106 (1957) 874.

[2] A. Marini, C. Hogan, M. Grüning, D. Varsano, Comp. Phys. Comm. 180 (2009) 1392.

[3] A. Castro et al., Phys. Stat. Sol. B 243 (2006) 2465.

[4] V. Mauchamp et al. Phys. Rev. B. 77 (2008) 045117.

[5] E. D. Palik, Handbook of Optical Constants of Solids, Academic Press, San Diego, 1998.

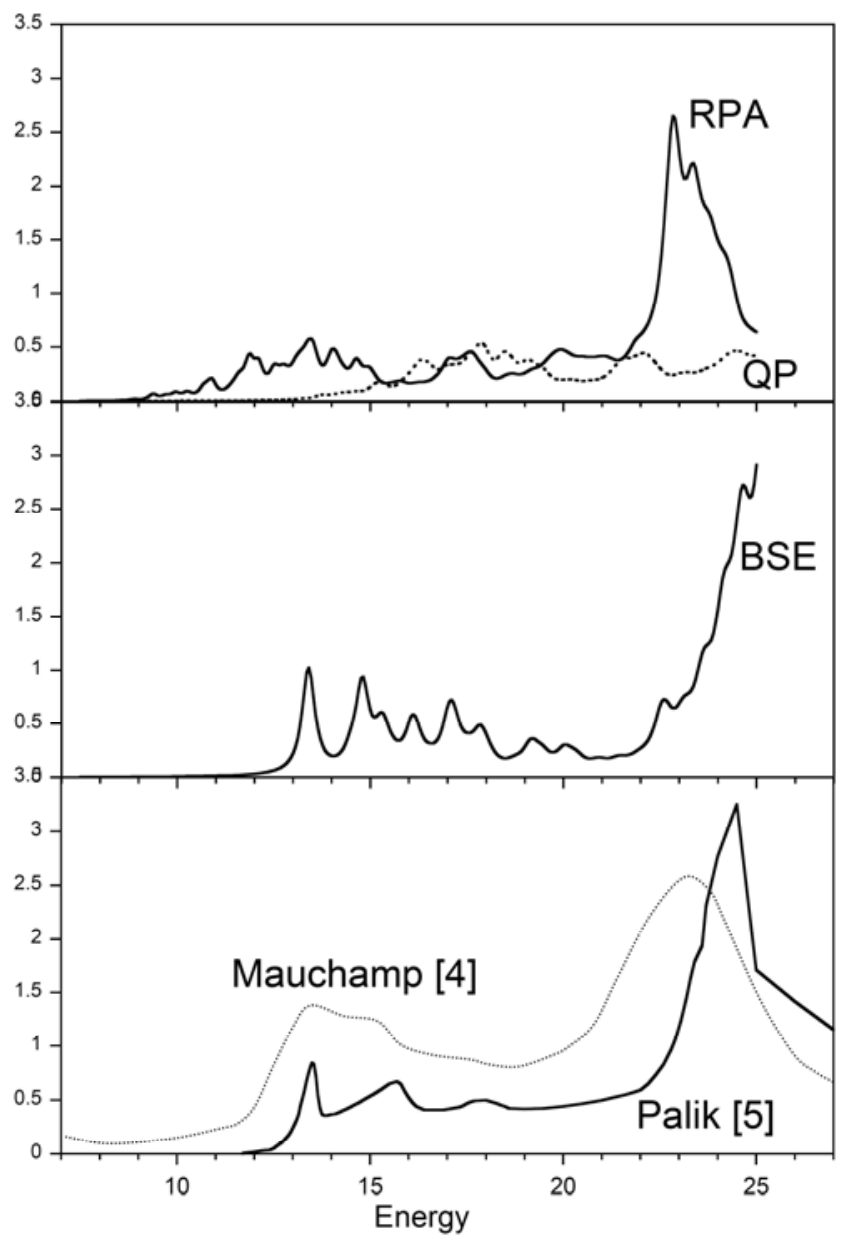

FIG. 1. EELS spectra for LiF calculated using the random phase approximation (RPA), including quasiparticle corrections $(\mathrm{QP})$ and with full excitonic effects and quasiparticle corrections using the Bethe-Salpeter equation (BSE). These are compared to the experimental EELS from Mauchamp [4] and the expected EELS based on the optical constants available in Palik [5]. The first sharp peak is an exciton peak which is only correctly predicted using the BSE.

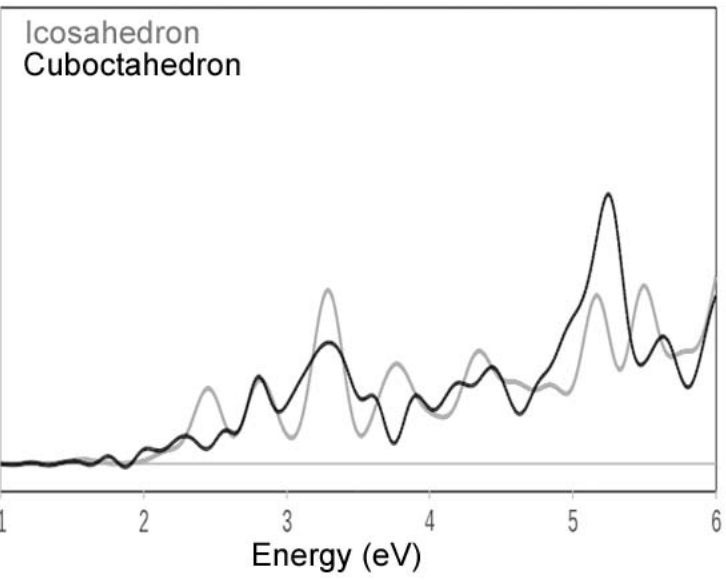

FIG. 2. Optical absorption spectra for two different atomic arrangements of a cluster of 55 gold atoms. There are significant differences between the two spectra. 